\title{
Notes
}

Hautahi Kingi*

\section{Welfare Analysis in an Extended \\ Harris-Todaro Model: An Application of the Atkinson Theorem}

DOI 10.1515/bejte-2015-0132

\begin{abstract}
I analyze the welfare effects of a policy of modern sector enlargement (MSENL), and a policy of increasing the efficiency of on-the-job search from the urban informal sector (IEOS) in a generalized Harris-Todaro model. I show that MSENL causes a Lorenz worsening of the income distribution and IEOS causes a Lorenz improvement. In a rare direct application of the Atkinson theorem, I conclude that MSENL decreases social welfare and IEOS increases social welfare for all anonymous, increasing and Schur-concave social welfare functions.
\end{abstract}

Keywords: Lorenz curves, segmented labor markets, inequality, welfare JEL Classification: D3, D6, J6, 02

\section{Introduction}

Most people in the developing world derive all their income from employment (World Bank 2012). The structure of labor markets, and the policies enacted within them, therefore dramatically affect the lives of the poor. The multi-sector labor market model of Harris and Todaro (1970) (HT) remains the basic framework for a vast literature devoted to analyzing labor market policies in developing economies. These analyses primarily focus on wages and unemployment, while ignoring the welfare consequences of labor market policies. One reason for this oversight is that it is generally difficult, within such models, to draw robust welfare conclusions that do not critically depend on the specific welfare criterion adopted (Temple 2005). For example, in one of the few welfare economic analyses in this literature, Fields (2005) shows that the welfare consequences of various labor market policies are ambiguous even when restricting the analysis to abbreviated welfare functions.

*Corresponding author: Hautahi Kingi, Cornell University, Ithaca, NY 14853-0001, USA, E-mail: hrk55@cornell.edu 
In this article, I derive welfare conclusions that are both unambiguous and robust to alternative specifications of welfare criteria. I do so by exploiting the classic Atkinson (1970) welfare theorem, which specifies conditions under which one can make welfare statements for an extremely broad class of social welfare functions. The usefulness of this theorem is generally limited by its lack of applicability because its conditions are seldom satisfied in practice (Dutta 2002). Thus, the direct application of the Atkinson (1970) theorem can itself be considered an additional contribution of this article.

I consider the generalized HT framework of Fields (1989), which incorporates two of the more important subsequent extensions in the literature informal sector dualism and on-the-job search. Within this framework, I analyze two labor market policies. The first, modern sector enlargement (MSENL), was the primary policy considered by HT and is now the benchmark policy for analyzing and comparing the structure of labor market models within the literature. The second, increasing the efficiency of on-the-job search from the urban informal sector (IEOS), is a novel policy enabled by informal sector dualism and on-the-job search.

I demonstrate that MSENL causes a Lorenz worsening of the income distribution and IEOS causes a Lorenz improvement. This is a novel result in itself given that policies within HT frameworks do not generally result in income distributions with non-intersecting Lorenz curves (Temple 2005). I also demonstrate that both MSENL and IEOS do not alter the mean of the income distribution. These properties satisfy the strict conditions of the Atkinson (1970) theorem, which I then invoke to conclude that MSENL reduces social welfare and IEOS improves social welfare for all anonymous, increasing and Schur-concave social welfare functions.

The article proceeds as follows. In Section 2, I describe the salient features of the extended Harris-Todaro model of Fields (1989). I conduct the welfare analysis in Section 3 and conclude in Section 4.

\section{Model}

Fields (1989) defines three employment sectors - the urban modern sector $(M)$, the urban traditional sector $(T)$, and the agricultural sector $(A)$. The urban modern sector pays the highest wage, $W_{M}$, which is exogenously set above the market clearing level by a combination of institutional and market forces. Thus, all workers aspire to jobs in this sector, but employment is fixed at a level $E_{M}$. Workers may elect to search for these jobs by opting for one of three search strategies. 
Strategy I is identical to the Harris and Todaro (1970) (HT) strategy of directing all effort toward search while being openly unemployed. Strategy II is to search part time while accepting low-wage employment in the land-abundant agricultural sector at a fixed wage $W_{A}$. Strategy III is to search part time from the urban traditional sector, while accepting low-wage employment at an endogenous wage $W_{T}$, assuming that a fixed amount of total earnings in the traditional sector, $Q_{T}$, is allocated evenly across workers, $L_{T}$, such that $W_{T}=\frac{Q_{T}}{L_{T}}$.

The probability of finding a job using strategy $I$ is $\pi$, which is determined endogenously and described in eq. [1] below. The expected earnings from this strategy are therefore $V_{I}=\pi W_{M}$. Workers who adopt search strategy $I I$ face a reduced probability $\theta \pi$ of obtaining a high-wage job, where $1>\theta>0$, because such workers can only devote part of their time to searching while maintaining agricultural employment. Expected earnings from strategy $I I$ are $V_{I I}=W_{M} \theta \pi+W_{A}(1-\theta \pi)$. Workers who opt for strategy III face a probability $\varphi \pi$ of finding a job in the urban modern sector. The corresponding expected earnings are $V_{I I I}=W_{M} \varphi \pi+W_{T}(1-\varphi \pi)$. It is assumed that $1>\varphi>\theta>0$ because the geographical distance between the agricultural and urban sector means that agricultural workers experience substantial inconvenience when searching for a job compared to traditional workers located in urban areas. The HT model is obtained within this framework by removing the urban traditional sector $\left(Q_{T}=0\right)$ and on-the-job search $(\varphi=\theta=0)$.

Denoting the number of workers electing search strategy $i \in\{I, I I, I I I\}$ by $L_{i}$, the probability of finding a job using strategy $I$ is defined as

$$
\pi=\frac{E_{M}}{L_{I}+\theta L_{I I}+\varphi L_{I I I}}
$$

The probability of finding a modern sector job is a function of the ex-ante number of workers who initially choose each search strategy, rather than the resulting ex-post allocations of workers across the three employment sectors. The relationships between the ex-post and ex-ante allocations are described by eqs [2]-[5].

$$
\begin{aligned}
& L_{M}=E_{M}=\pi\left(L_{I}+\theta L_{I I}+\varphi L_{I I I}\right) \\
& L_{A}=L_{I I}(1-\theta \pi) \\
& L_{T}=L_{I I I}(1-\varphi \pi) \\
& U=L_{I}(1-\pi)
\end{aligned}
$$

Equation [2] demonstrates that modern sector employment $L_{M}$ is fixed at $E_{M}$, which is comprised of the successful job searchers from each of the search strategies. Equations [3]-[5] demonstrate that agricultural employment $L_{A}$, urban traditional sector employment $L_{T}$, and open unemployment $U$ are 
determined by the number of workers who are unsuccessful in obtaining highwage employment from search strategy II, III and I, respectively. The ex-ante and ex-post labor market clearing conditions are given by

$$
L=L_{I}+L_{I I}+L_{I I I}=L_{M}+L_{T}+L_{A}+U
$$

The $L$ workers in the economy initially allocate themselves across the three search strategies. Once the labor market clears, workers find themselves in one of the four employment states. Search strategies are chosen in order to maximize expected earnings. An interior solution to this problem therefore requires that all three search strategies yield the same expected value. That is,

$$
V_{I}=V_{I I}=V_{I I I}
$$

The model, which is represented by eqs [1]-[7], can be solved for the following ex-post allocations of workers in terms of exogenous variables. ${ }^{1}$

$$
\begin{gathered}
L_{T}=\frac{Q_{T}(1-\theta)}{W_{A}(1-\varphi)}-\frac{Q_{T}(\varphi-\theta)}{W_{M}(1-\varphi)} \\
L_{A}=\frac{L}{1-\theta\left(1-\frac{W_{A}}{W_{M}}\right)}-\frac{E_{M} W_{M}}{W_{A}}-\frac{Q_{T}}{W_{A}} \\
U=L-L_{T}-L_{A}-E_{M}
\end{gathered}
$$

Total income in the economy is given by

$$
I=Q_{T}+L_{A} W_{A}+E_{M} W_{M}
$$

\section{Welfare Analysis}

I evaluate the welfare effects of a policy of modern sector enlargement (MSENL) and a policy of increasing the efficiency of on-the-job search from the urban informal sector (IEOS) by comparing equilibrium income distributions before and after the application of each policy. MSENL is modeled as an increase in $E_{M}$, while IEOS is modeled as an increase in $\varphi$.

In general, making unambiguous welfare comparisons depends critically on which specific welfare criterion is adopted. Thereom 1, proposed by Atkinson (1970) and later extended by Dasgupta, Sen, and Starrett (1973), states that under certain conditions, however, the welfare of one income distribution may be ranked relative to another for a broad range of social welfare functions.

1 The derivations can be found in the Appendix. 
Theorem 1: Let $X$ and $Y$ be two income distributions with equal means. Let $\mathbb{W}$ denote the class of anonymous, increasing and Schur-concave social welfare functions. Then, $X$ Lorenz dominates $Y$ if and only if $w(X)>w(Y)$ for all $w \in \mathbb{W}$.

Theorem 1 facilitates the welfare analysis in two ways. First, it reduces an analysis of welfare to one of inequality. If workers derive utility solely from income, which is implied by the search strategy behavior described by eq. [7], then an income distribution that Lorenz dominates another with the same mean is not only more equal, but better in terms of welfare. Thus, Theorem 1 ensures that the income distribution provides sufficient information to make welfare inferences.

Second, Theorem 1 ensures the robustness of any welfare implications, because the great majority of accepted social welfare functions are included within $\mathbb{W}$. Anonymity simply requires that all workers are treated identically regardless of their income level. The "increasing" property ensures that social welfare increases whenever one worker's earnings increase, holding other workers earnings constant. Finally, Schur-concavity, - a weaker condition than concavity - ensures that the "transfer principle" of Pigou (1912) and Dalton (1920) is satisfied. That is, a welfare function registers an increase in well-being when income is transferred from a richer to a poorer worker without reversing the ranking of each. I address the prerequisite of Theorem 1 in Proposition 1.

Proposition 1: MSENL and IEOS do not affect the mean of the income distribution.

Proof: The model emits explicit equations for labor allocations and earnings. I can therefore assess the impact of MSENL and IEOS by evaluating derivatives with respect to $E_{M}$ and $\varphi$, respectively. Because population is fixed at $L$, it is sufficient to show that total income, $I$, is unchanged. It follows from eq. [11] that

$$
\begin{aligned}
\frac{\partial I}{\partial E_{M}} & =\frac{\partial L_{A}}{\partial E_{M}} W_{A}+W_{M} \\
\frac{\partial I}{\partial \varphi} & =\frac{\partial L_{A}}{\partial \varphi}
\end{aligned}
$$

because $Q_{T}, W_{A}$ and $W_{M}$ are exogenously fixed. Equation [9] implies that $\frac{\partial L_{A}}{\partial E_{M}}=-\frac{W_{M}}{W_{A}}$ and $\frac{\partial L_{A}}{\partial \varphi}=0$. Respectively substituting these expressions into [12] and [13] yields $\frac{\partial I}{\partial E_{M}}=0$ and $\frac{\partial I}{\partial \varphi}=0$ as required.

Proposition 1 demonstrates that MSENL and IEOS reallocate workers and earnings across sectors in a manner that is neutral in terms of total income, thereby allowing Theorem 1 to be applied. It therefore remains to determine whether the labor market policies yield non-intersecting Lorenz curves. 


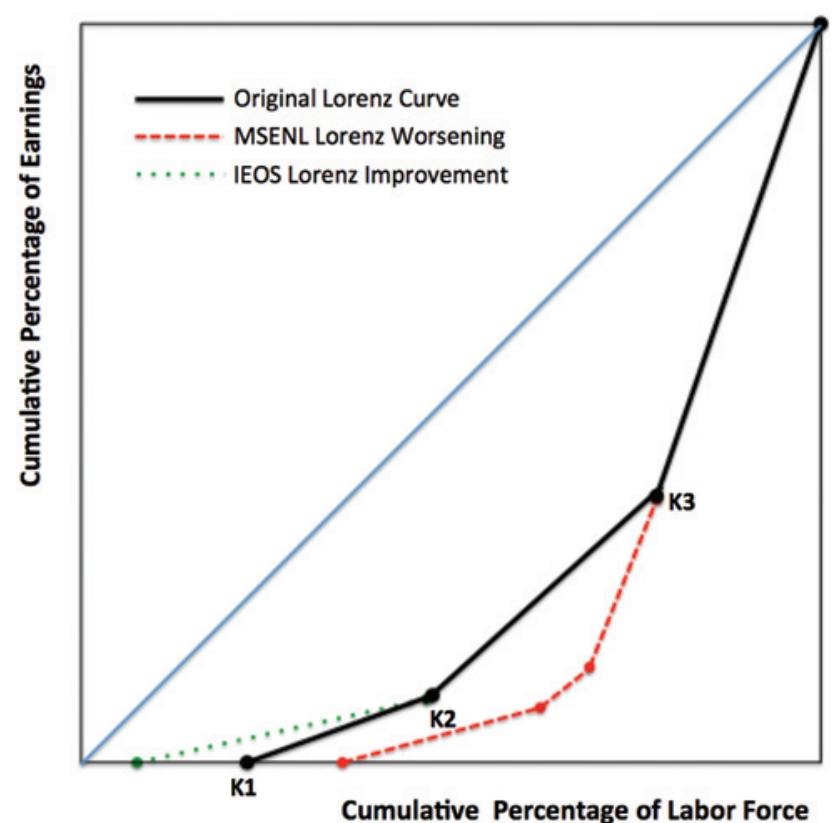

Figure 1: Lorenz curve comparisons.

Proposition 2: MSENL causes a Lorenz worsening.

Proof: Workers fall into one of four employment categories. A Lorenz curve therefore consists of four piecewise linear segments, each characterized by the position of three kink points as shown in Figure 1. Without loss of generality, I normalize $I=1$ and $L=1$ so that the coordinates of the kink points are $K_{1}=(U, 0)$, $K_{2}=\left(U+L_{T}, Q_{T}\right)$ and $K_{3}=\left(1-E_{M}, 1-W_{M} \cdot E_{M}\right)$. Equations [8]-[10] imply

$$
\frac{\partial U}{\partial E_{M}}=\frac{W_{M}}{W_{A}}-1>0, \frac{\partial L_{T}}{\partial E_{M}}=0
$$

The increase in unemployment shifts $K_{1}$ to the right which also shifts $K_{2}$ to the right because $L_{T}$ is unchanged. The vertical position of $K_{2}$ is unaltered because $Q_{T}$ is exogenously fixed. The increase in $E_{M}$ directly shifts $K_{3}$ down and to the left. The resulting Lorenz curve following MSENL lies partly below and never above the original Lorenz curve as depicted by the dashed red line in Figure 1. This is a Lorenz worsening.

Proposition 3: IEOS causes a Lorenz improvement. 
Proof: Equations [8]-[10] imply

$$
\frac{\partial U}{\partial \varphi}=-\frac{Q_{T}(1-\theta)}{(1-\varphi)^{2}}\left[\frac{1}{W_{A}}-\frac{1}{W_{M}}\right]<0, \frac{\partial L_{T}}{\partial \varphi}=\frac{Q_{T}(1-\theta)}{(1-\varphi)^{2}}\left[\frac{1}{W_{A}}-\frac{1}{W_{M}}\right]>0, \frac{\partial L_{A}}{\partial \varphi}=0
$$

The decrease in unemployment shifts $K_{1}$ to the left. Because $E_{M}$ and $L_{A}$ are unaffected, $U+L_{T}=L-E_{M}-L_{A}$ is unchanged and therefore $K_{2}$ remains in its horizontal position. $K_{2}$ also remains in its vertical position because $Q_{T}$ is exogenously fixed. Fixed levels of $E_{M}$ and $W_{M}$ ensure that $K_{3}$ is unaltered. The resulting Lorenz curve following IEOS lies partly above and never below the original Lorenz curve as depicted by the dotted green line in Figure 1. This is a Lorenz improvement.

Propositions 2 and 3 demonstrate that both policies produce strict Lorenz orderings. In isolation, these results imply that MSENL increases inequality and IEOS decreases inequality. When combined with Theorem 1 and Proposition 1, however, the findings also have normative significance. Theorem 2, which is the main result of this article, follows directly from Theorem 1 and Propositions 1-3.

Theorem 2: For all anonymous, increasing and Schur-concave social welfare functions, MSENL decreases social welfare and IEOS increases social welfare.

\section{Conclusion}

Within the context of the generalized Harris-Todaro model proposed by Fields (1989), I demonstrate that both MSENL and IEOS do not alter the mean of the income distribution. I then show that MSENL causes a Lorenz worsening and IEOS causes a Lorenz improvement. These properties allow me to draw robust welfare conclusions by exploiting Atkinson's (1970) classic theorem, which demonstrates that Lorenz orderings coincide with welfare orderings for a very broad class of welfare functions. I conclude that a policy of MSENL therefore reduces social welfare while a policy of IEOS improves social welfare.

This article makes three contributions. First, it extends the work of Fields (2005), who performs welfare economic analyses within the basic HT framework, to the more complex multi-sector labor market model of Fields (1989). Second, it widens the scope of policy analysis to include welfare effects that are robust to all anonymous, increasing and Schur-concave social welfare functions. Third, 
and relatedly, it provides what is to my knowledge the first direct application of the Atkinson (1970) theorem within a multi-sector labor market model.

Acknowledgments: This work originated as a term paper for a Cornell University Development Economics course taught by Gary Fields. I would like to thank him for the inspiration. I am also grateful for the insightful comments provided by Kyle Rozema and two anonymous referees.

\section{Appendix}

\section{Derivation of Eqs [8]-[9]}

Equation [7] implies that

$$
\begin{aligned}
& (1-\theta) \pi W_{M}=W_{A}(1-\theta \pi) \\
& (1-\varphi) \pi W_{M}=W_{A}(1-\varphi \pi)
\end{aligned}
$$

after substituting in the values of each of the search strategies $V_{i}$. These can be rearranged to yield

$$
\begin{aligned}
& \frac{1}{\pi}=(1-\theta) \frac{W_{M}}{W_{A}}+\theta \\
& \frac{1}{\pi}=(1-\varphi) \frac{W_{M}}{W_{T}}+\varphi
\end{aligned}
$$

Equating the right hand sides of [16] and [17] yields

$$
(1-\theta) \frac{W_{M}}{W_{A}}+\theta=(1-\varphi) \frac{W_{M}}{W_{T}}+\varphi
$$

which can be rearranged to yield eq. [8] after substituting in for $W_{T}=\frac{Q_{T}}{L_{T}}$.

The definition of $\pi$ in eq. [1] can be rearranged to yield

$$
\begin{gathered}
\pi\left(L_{I}+\theta L_{I I}+\varphi L_{I I I}\right)=E_{M} \\
\Rightarrow \pi\left(\frac{U}{1-\pi}+\theta \frac{L_{A}}{1-\theta \pi}+\varphi \frac{L_{T}}{1-\varphi \pi}\right)=E_{M} \\
\Rightarrow \pi\left(\frac{L-L_{A}-L_{T}-E_{M}}{1-\pi}+\theta \frac{L_{A}}{1-\theta \pi}+\varphi \frac{L_{T}}{1-\varphi \pi}\right)=E_{M} \\
\Rightarrow \frac{\pi}{1-\pi}\left(L-E_{M}-\frac{W_{A}}{W_{M} \pi} L_{A}-\frac{W_{T}}{W_{M} \pi} L_{T}\right)=E_{M}
\end{gathered}
$$




$$
\begin{aligned}
& \Rightarrow L_{A}=\frac{W_{M} \pi}{W_{A}} L-\frac{Q_{T}}{W_{A}}-\frac{W_{M}}{W_{A}} E_{M} \\
& \Rightarrow L_{A}=\frac{W_{M}}{W_{A}} \frac{1}{(1-\theta) \frac{W_{M}}{W_{A}}+\theta} L-\frac{Q_{T}}{W_{A}}-\frac{W_{M}}{W_{A}} E_{M} \\
& \Rightarrow L_{A}=\frac{L}{1-\theta\left(1-\frac{W_{A}}{W_{M}}\right)}-\frac{E_{M} W_{M}}{W_{A}}-\frac{Q_{T}}{W_{A}}
\end{aligned}
$$

which is [9].

\section{References}

Atkinson, A. 1970. "On the Measurement of Inequality." Journal of Economic Theory 2:244-63. Dalton, H. 1920. "The Measurement of the Inequality of Incomes." Economic Journal 30:348-61.

Dasgupta, P., A. Sen, and D. Starrett. 1973. "Notes on the Measurement of Inequality." Journal of Economic Theory 6:180-7.

Dutta, B. 2002. "Inequality, Poverty and Welfare." In Handbook of Social Choice and Welfare, Volume 1, edited by K. Arrow, A. Sen, and K. Suzumura, 597-633. North Holland, Amsterdam: Elsevier, chapter 12, 1 edition.

Fields, G. 1989. "On-the-Job Search in a Labor Market Model: Ex Ante Choices and Ex Post Outcomes." Journal of Development Economics 30:159-78.

Fields, G. 2005. "A Welfare Economic Analysis of Labor Market Policies in the Harris-Todaro Model." Journal of Development Economics 76:127-46.

Harris, J., and M. Todaro. 1970. "Migration, Unemployment \& Development: A Two-Sector Analysis." American Economic Review 60:126-42.

Pigou, A. 1912. "Wealth and Welfare." London: MacMillan.

Temple, J. 2005. "Growth and Wage Inequality in a Dual Economy." Bulletin of Economic Research 57:145-69.

World Bank. 2012. World Development Report 2013: Jobs. Washington, DC: World Bank. 